\title{
Performance evaluation of interference aware topology power and flow control channel assignment algorithm
}

\author{
Jatinder Singh Saini' ${ }^{1}$, Balwinder Singh Sohi ${ }^{2}$ \\ ${ }^{1}$ I. K. Gujral Punjab Technical University, India \\ ${ }^{2}$ Chandigarh Group of Colleges, India
}

\begin{tabular}{l} 
Article Info \\
\hline Article history: \\
Received Nov 4, 2018 \\
Revised May 7, 2019 \\
Accepted Nov 22, 2019 \\
\hline
\end{tabular}

Keywords:

Channel assignment

Flow control

Power control

Topology control

WiMesh

\begin{abstract}
Multi-Radio Multi-Channel Wireless Mesh Network (MRMC-WMN) has been considered as one of the key technology for the enhancement of network performance. It is used in a number of real-time applications such as disaster management system, transportation system and health care system. MRMC-WMN is a multi-hop network and allows simultaneous data transfer by using multiple radio interfaces. All the radio interfaces are typically assigned with different channels to reduce the effect of co-channel interference. In MRMC-WMN, when two nodes transmit at the same channel in the range of each other, generates co-channel interference and degrades the network throughput. Co-channel interference badly affects the capacity of each link that reduces the overall network performance. Thus, the important task of channel assignment algorithm is to reduce the co-channel interference and enhance the network performance. In this paper, the problem of channel assignment has been addressed for MRMC-WMN. We have proposed an Interference Aware, Topology, Power and Flow Control (ITPFC) Channel Assignment algorithm for MRMC-WMN. This algorithm assignes the suitable channels to nodes, which provides better link capacity and reduces the co-channel interference. In the previous work performance of the proposed algorithm has been evaluated for a network of 30 nodes. The aim of this paper is to further evaluate the performance of proposed channel assignment algorithm for 40 and 50 nodes network. The results obtained from these networks show the consistent performance in terms of throughput, delay, packet loss and number of channels used per node as compared to LACA, FCPRA and IATC Channel Assignment algorithms.
\end{abstract}

Copyright @ 2020 Institute of Advanced Engineering and Science. All rights reserved.

Corresponding Author:

Jatinder Singh Saini,

I. K. Gujral Punjab Technical University,

Jalandhar, Punjab, India.

Email: sainijatinder@gmail.com

\section{INTRODUCTION}

Multi-Radio Multi-Channel Wireless Mesh Network (MRMC-WMN) is a promising technology which provides a solution for wide areas to offer wireless connectivity without using wired infrastructure. MRMC WMN facilitates a variety of emerging and industrial application regions e.g. local community networks, broadband home networking and disaster management [1]. WMNs are recognized by efficient, self-organizing, self-healing characteristics and are made up of a huge number of mesh nodes. Mesh nodes consist of mesh routers, mesh gateways and mesh clients as depicts in Figure 1 [2]. Mesh gateways are associated with the wired backbone to provide network services to the mesh clients. Mesh routers establish the connection with mesh clients and redirect the data towards the destination node. Every node operates on different frequency channels to manage the co-channel interference between nodes [3]. MRMC-WMN works in two modes such as Ad-hoc mode and infrastructure mode. As shown in Figure 1 Mesh router domain and 
Mesh client domain works in Ad-hoc mode. All the nodes in Ad-hoc mode are directly associated to each other. They form peer-to-peer connections in the network and no any intermediate node is required toestablish the connection. In infrastructure mode, an intermediate node is required for data transmission. As shown in Figure 1 mesh clients are connected to mesh routers in infrastructure mode.

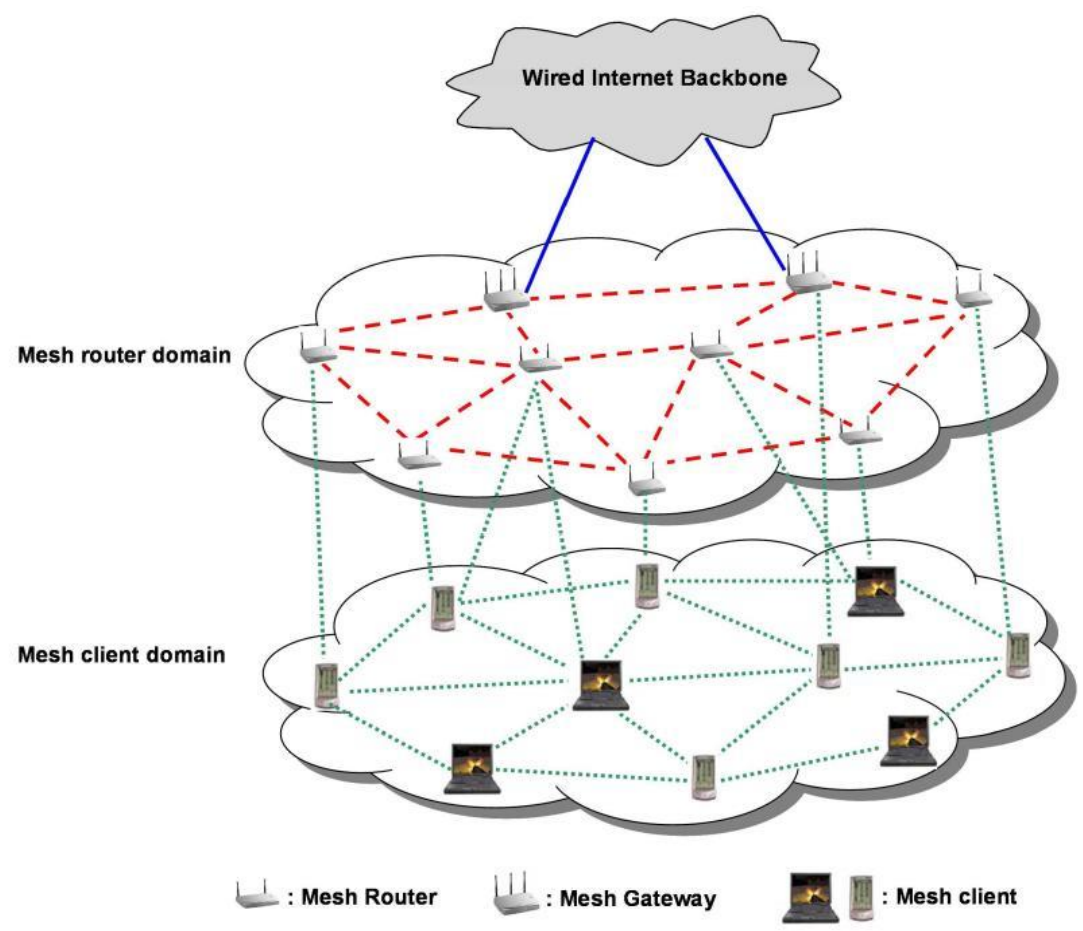

Figure 1. MRMC WMN architecture

As the mesh nodes are equipped with cost-effective multiple network interface cards (NICs), nodes can transfer and receive data at the same time which enhance the speed of data transmission. Due to the availability of limited numbers of non-overlapping channels, co-channel interference occurs in the network [4]. Co-channel interference reduces the capacity of each link which leads toward lower network performance. To minimize the co-channel interference in MRMC-WMN effective channel assignment algorithm is needed. Channel assignment is the method which ensures that all the interfering nodes are assigned with distinct channels. There are some important factors which need to consider during the channel assignment i.e., interference, power control, topology control and flow control.

Topology control plays an important role to attain the required topology. By turning on/off node's NICs and creating the new links, topology control makes the communication backbone of WMN. The network topology can logically change the network structure to enhance the network functions such as power effectiveness, link connections, flexibility, capacity enhancement and interference elimination etc. [5]. In MRMC-WMN power control manages the interference range and transmission range of every node. To manage the interference and network connectivity the transmission power of every node must be controlled to the essential level. Effective power control minimizes co-channel interference among nodes and improves channel reuse [6]. Flow control in MRMC-WMN ensures that every node obtains the required link capacity. Due to co-channel interference capacity of each link has been reduced. The inadequate link capacity slows down the transmission speed and degrades the overall network performance [7]. MRMC WMN attains optimum network throughput and the higher end to end data rate by controlling the flow of every link.

In this paper, Interference Aware, Topology, Power, Flow Control (ITPFC) Channel Assignment algorithm has been proposed for MRMC WMN. In the previous work, the performance of the proposed algorithm has been evaluated for a network of 30 nodes [8]. It is necessary to test the algorithm for different and large network. Because, an algorithm only completes when, it is tested under scalable networks. So, two new networks of 40 and 50 nodes have been designed by installing the nodes on random locations. Then, the performance of proposed algorithm has been evaluated on these networks. To design and examine the proposed channel assignment algorithm simulation test-bed has been designed using NS-3 based WiMesh 
simulation tool. Firstly, networks of 40 and 50 nodes have been implemented using Interference Aware Topology Control Channel Assignment algorithm. After this, ITPFC Channel Assignment algorithm has been applied on the network. First of all the algorithm dynamically control the transmission power of each node. The algorithm controls the transmission power of each node at the required level and minimizes the co-channel interference. Then, algorithm assures that if any link caused by co-channel interference is not receiving adequate link capacity then reassign the channel with least interference channel and meets the requirements. Proposed ITPFC Channel Assignment algorithm has been compared with existing channel assignment algorithms in terms of throughput, delay, packet loss and number of channels used per node. It has been seen that proposed algorithm perform better as compared to Load-Aware Channel Assignment Algorithm (LACA) [9], Flow-based Channel and Rate Assignment Algorithm (FCPRA) [10], and Interference-Aware Topology Control Channel Assignment Algorithm (IATC) [11]. Rest of the paper is organized as follows. Section II includes related work to channel assignment. In section III, the network model and the proposed algorithm has been discussed. Section IV includes the results and discussions. Finally, Section V concludes the paper.

\section{RELATED WORK}

In [12] authors introduced the load balancing technique to solve the problem of multicast routing and channel assignment. It creates the multicast weighted conflict graph and based on the link's weight measures the interference degree of each node. Simulation results determine that this technique reduces the interference among nodes and computational complexity. Authors in [13] proposed a routing metric that provides information about link availability, available link bandwidth, quality of link and data loss rate. This metric balanced the traffic load on each link and reduces the interference as well as avoids the bottleneck formation in the network. This routing metric achieves higher throughput and support network scalability as compared to the existing routing algorithms.

In [14] authors proposed Traffic-aware Channel Assignment algorithm that obtains the traffic information of the network nodes and assigns an efficient channel. Based on mathematically model and realtime experiments the effectiveness of the proposed algorithm has been measured and it is found that the proposed algorithm has better network throughput as compared to existing schemes. Authors in [15] proposed CSMA-aware Static Channel Assignment algorithm that assigns collision free channel. Based on CSMA-aware interference model and link limitation strategy proposed algorithm reduces the interference among nodes. Simulation results prove that the proposed algorithm has better traffic performance in term of lower interference as compared to existing algorithms. In [16] the authors developed a Minimum Interference Channel Assignment algorithm for multicast WMN. Based on the interference factor the proposed algorithm identifies the interference relationship between multicast tree nodes and assigns less interference channels. Simulation results depict that proposed algorithm achieved higher throughput and lower end-to-end delay as compared to Multi-channel Multicast Channel Assignment algorithm. Authors in [17] studied an optimization model for MRMC-WMN to minimize the spectrum requirements while altering the traffic demands. This model takes care of various important aspects such as channel assignment, power control, routing and interference model. Simulation experiments determine the significant saving in spectrum usage and better results in terms of offered load, radio patterns and network density. In [18] the authors proposed an optimization framework based on linear programming to study the relationship between throughput and energy consumption. Authors used the physical interference model to continuously control the transmission power of each node. Numerical results show that the proposed model achieves higher throughput and lower power consumption.

In [19] the authors proposed the channel assignment algorithm based on link layer protocol and learning automata to enhance the performance of WMNs. Learning automata intelligently assign the channels to avoid the interference among nodes. Results describe that proposed algorithm has better performance in terms of packet drop, delay average goodput, jitter and energy usage as compared to Ad-hoc on Demand Distance Vector protocol.Authors in [20] presented the survey of various channel assignment algorithms for MRMN-WMNs and provide the latest finding of numerous researches. Authors categorized the channel assignment algorithms into sequential multicast routing \& channel assignment algorithm and joint multicast routing \& channel assignment algorithm. These algorithms are further divided into centralized and distributed channel assignment algorithms and illustrated the advantages and disadvantages of these algorithms. In [21] authors proposed multiple access scheduling in MRMC based on the Latin Square to enhance the network performance. This algorithm based on interference detail collected from the network, creates the inter-cluster and intra-cluster in the network and map the clustering structure to radios and channels. Simulation results show that the proposed algorithm performs better than the IEEE 802.11 standards and other channel access control protocols. 
In [22] author proposed Energy Efficient Channel Assignment and Routing Algorithm-Traffic Demands algorithm for multi-radio WMNs. The aim of this algorithm is to minimize the energy consumption and total utilization of the entire collision domain. Simulation results prove that the proposed algorithm performs better as compared to the existing work. The authors in [23] proposed an enhanced Topologycontrolled Interference-aware Channel-assignment (e-TICA) algorithm to solve the problem of unforeseeninterference using two-way interference-range edge coloring model. This algorithm examines the links that come under interference range and identify the channel being used to concerned nodes. It ensures that nodes in interference range are not assigned with the same channel. Simulation results show that e-TICA performs better as compared to TICA and CCA in term of fairness ratio among flows without reducing the network throughput. In [24] authors proposed a joint channel assignment and flow allocation algorithm for MRMC-WMNs by using overlapped and partially overlapped channels. This algorithm considers important network parameters such as the path loss information, transmission power of each node, the signal to interference plus noise ratio at a node and the frequency response of the filters used in the transmitter and receiver. Proposed algorithm provides superior channel assignments and flow allocations using partially overlapped channels, without the need for any additional spectrum.

\section{NETWORK MODEL}

The proposed algorithm has been evaluated using two network scenarios. In the first scenario, 40 nodes have been deployed at random locations and the second scenario has been designed using 50 nodes as shown in Figure 2 (a) and (b). All the network nodes in both scenarios have been configured with three radio interfaces. Both scenarios are designed in $300 \mathrm{~m} \mathrm{X} \mathrm{600m} \mathrm{area.} \mathrm{All} \mathrm{the} \mathrm{nodes} \mathrm{are} \mathrm{configured} \mathrm{as} \mathrm{per}$ Table 1.

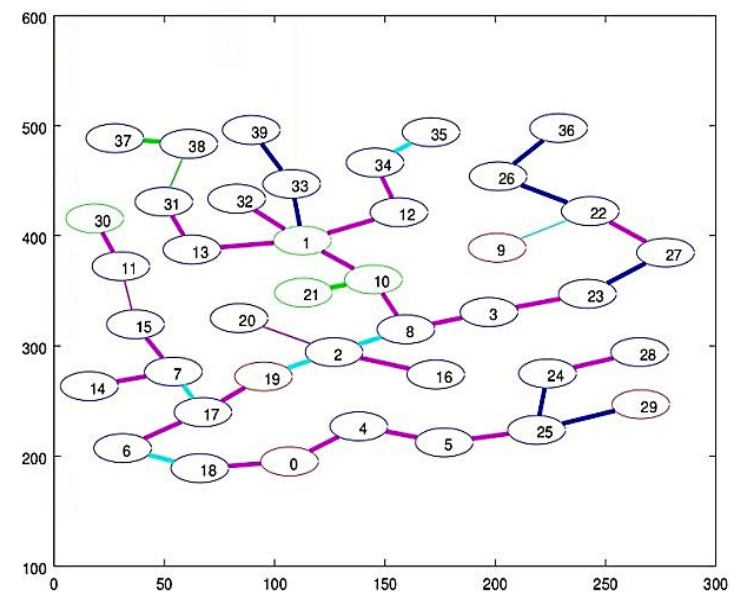

Figure 2a. Network topology of 40 nodes

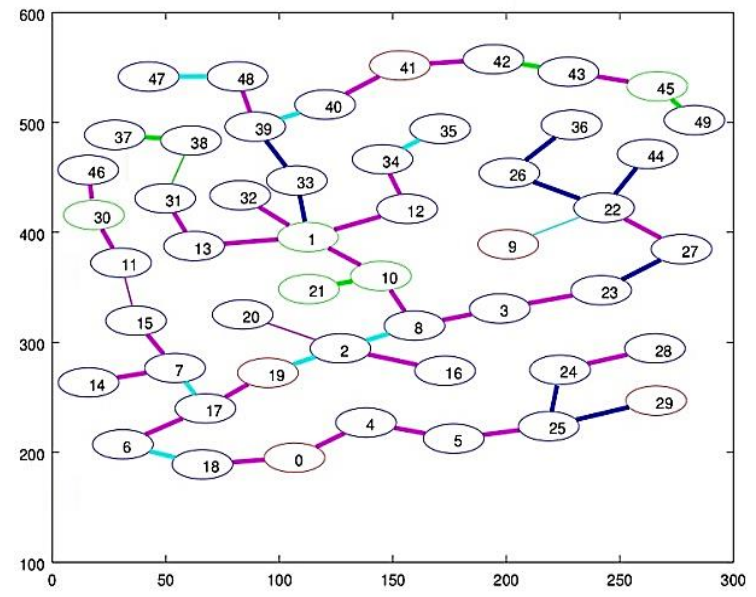

Figure 2b. Network topology of 50 nodes

Table 1. Node configuration

\begin{tabular}{ll}
\hline Description & Value \\
\hline Area & $300 \mathrm{~m} \mathrm{X} \mathrm{600m}$ \\
Simulation Time & $600 \mathrm{secs}$ \\
Routing Protocol & MPLS \\
Transmission Speed & $6 \mathrm{Mbps}$ \\
Interference Model & SINR \\
Receiving Threshold & $-46 \mathrm{dBm}$ \\
Aggregate Nodes & $4 / 5$ \\
Gateway Nodes & $4 / 5$ \\
No. of Channels & 11 \\
Traffic Type & On-Off \\
Flow Monitor Interval & $1 \mathrm{sec}$ \\
Transmission Gain & $1 \mathrm{dBi}$ \\
Receiver Gain & $1 \mathrm{dBi}$ \\
\hline
\end{tabular}


Simulation has been run for 600 seconds to accurately determine the performance of the proposed algorithm. The Multiprotocol Label Switching (MPLS) algorithm has been used as the routing algorithm. Initially, the transmission speed is set as $6 \mathrm{Mbps}$ and interference model is set as SINR. Aggregate nodes as well as gateway nodes are set as four and five for the network of 40 nodes and 50 nodes respectively. Receiving threshold value is set as $-46 \mathrm{dBm}$ and traffic type for data transmission in the network is used as On/Off. On/Off transmits data based on constant bit rate (CBR). Flow monitor interval is set as 1 second and transmitter gain, as well as receiver gain, is set as $1 \mathrm{dBi}$. All the available 11 channels in $2.4 \mathrm{GHz}$ band are used for channel assignment in the network.

The proposed algorithm has been designed in two stages. In the first stage, networks of 40 and 50 nodes have been designed using Interference Aware Topology Control channel assignment algorithm [11]. This algorithm assigns the channel with minimum interference among network nodes while considering the neighbor nodes. Using topology control this algorithm establishes the shorter and required links only.

In the second stage, ITPFC Channel Assignment algorithmhas been applied to assign best possible channel to nodes. This algorithm first, controls the transmission power of each node at the optimal level up to the neighbor node. Because MRMC-WMN is a multi-hop network and each node transfers the data to the neighbor node, towards the destination node. The algorithm calculates the distance between the concerned node $u$ and the neighbor node $v$. Then, the algorithm calculates the minimum transmission power, using the Friis transmission equation (1) [25], required between the nodes and compares with already assigned transmission power. In his equation Ptx is denoted as transmission power and Prx is denoted as receiving power. Gtx and Grx are represented as transmission gain and receiving gain respectively. $d$ is denoted as distance between node.

$$
P_{t x}=P_{r x}-G_{t x}-G_{r x}-20 \log _{10}\left(\frac{\lambda}{4 \pi d}\right)
$$

If the calculated transmission power is less than the assigned power then the algorithm reduces the transmission power of node $u$ and if calculated transmission power is greater than the assigned power then the algorithm increases the transmission power. If the transmission power is equal to the assigned power then no need to change anything. This algorithm tends to assign optimal transmission power for each node. The controlled transmission power leads toward the lower co-channel interference among nodes and improves the network performance.

Secondly, this algorithm ensures that each node obtains the required link capacity. The algorithm calculates the link capacity using equation (2) [26] as well as traffic flow between node $u$ and $v$ and compared the link capacity with traffic flow In this equation $W$ is denoted as bandwidth, $P$ and $N_{o}$ are represented as average power received and noise factor respectively. Iisdenoted as interference.

$$
C=W \log _{2}\left(1+\frac{P}{N_{0} W+I}\right)
$$

If traffic flow is greater than the link capacity then the algorithm verifies the assigned channels on different radios of the nodes against the number of NICs $(n)$ per node. The algorithm checks that, if channels of $u$ and $v$ nodes are less than the number of NICs $n$ then assign the channel $c$ to both nodes. If the channel of node $u$ is equal to the number of NICs $n$ and channels of node $v$ is less than number of NICs $n$ then selects the channel $c$ from node $u$ and assigns to node $v$. If channels of nodes $u$ and $v$ are equal to the number of NICs $n$ then use a common channel $c$ among nodes. Otherwise, select the channel $c 1$ from node $u$ and $c 2$ from node $v$, replace them with channel $c$ ' and assign to node $u$ and $v$. This algorithm reassigns the channel on the link that provides the required link capacity and improves the network throughput. Detailed step by step working of this algorithm is given in Algorithm 1. Performance of proposed ITPFC Channel Assignment algorithm has been evaluated in the next section by comparing the results in terms of throughput, delay, packet loss and number of channels used per node.

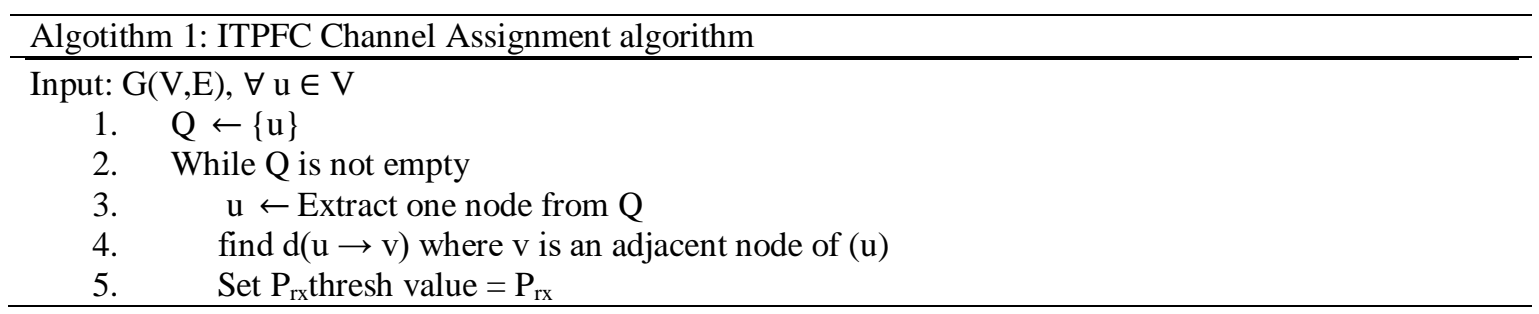




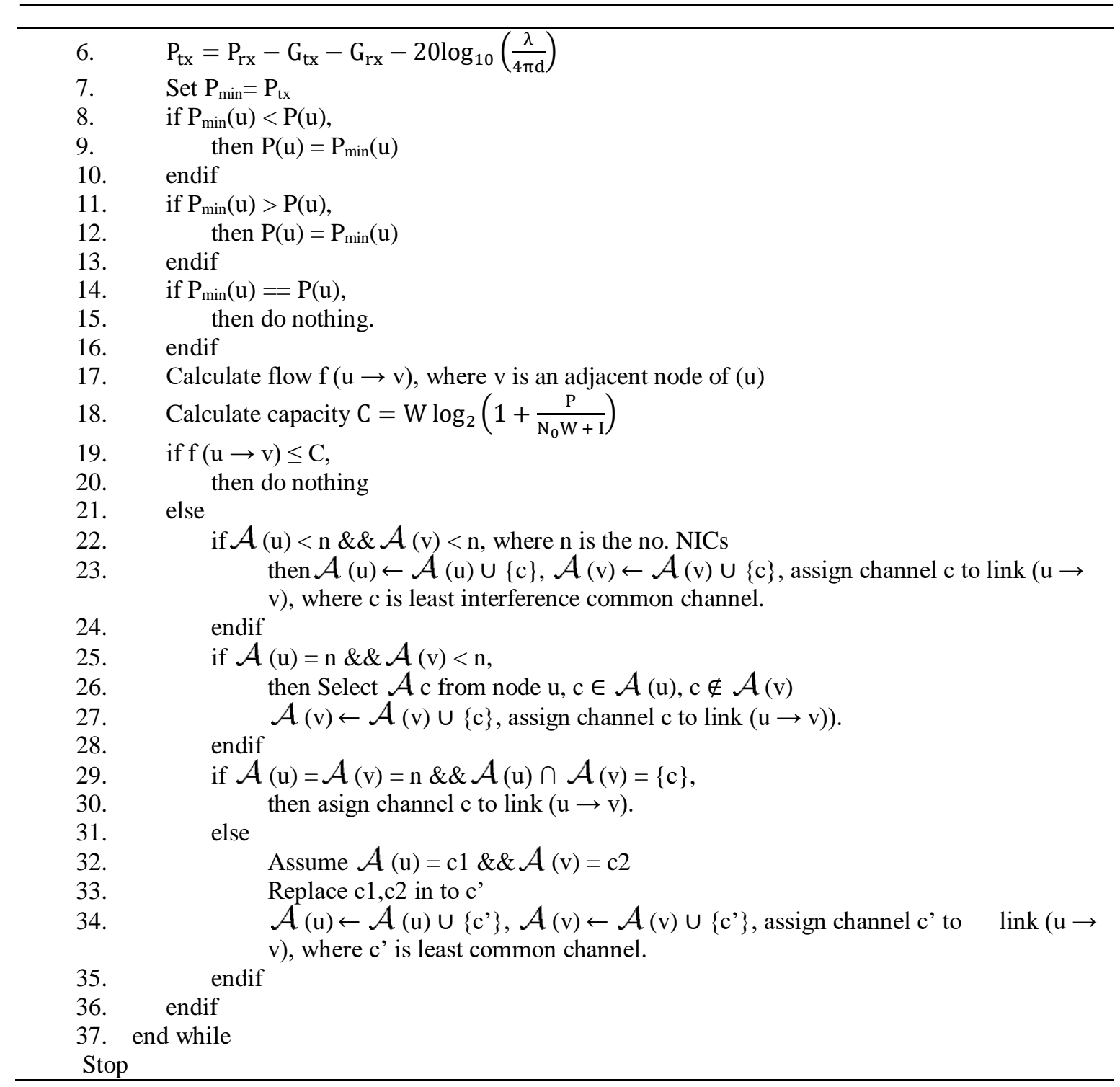

\section{RESULTS AND DISCUSSION}

To evaluate the effectiveness of proposed ITPFC Channel assignment algorithmresults are compared with Flow based Channel, Power and Rate Assignment Algorithm (FCPRA), Load Aware Channel Assignment Algorithm (LACA) and Interference Aware Topology Control Channel Assignment algorithm (IATC). Comparisons of above said algorithms are given below in two parts. Firstly, results are compared for the network of 40 nodes and secondly, comparison is done on 50 nodes network.

\subsection{Results comparison of $\mathbf{4 0}$ nodes network}

Figures 3, 4, 5 and Table 2 show the results obtained from 40 node's network in terms of throughput, delay, packet loss and number of channels used per node as compared to FCPRA, LACA and IATC Channel Assignment algorithms. Results illustrate the improved performance of the proposed ITPFC Channel assignment algorithm because the proposed algorithm reduces the co-channel interference and improves the link capacity of each node. When node achieves the suitable link capacity then it can easily transfer the data at the faster rate. The results of ITPFC Channel Assignment algorithm and LACA are very closely comparable in term of throughput and delay. But shows the significant improvement in packet loss and number of channels used per node in the network. As shown in Table 1, in ITPFC 56 radio interfaces are off while in IATC, LACA and FCPRA 45, 51 and 29 numbers of radio interfaces are offrepectively. The ITPFC Channel Assignment algorithm uses less number of radio interfaces to setup the communication links among nodes as compared to LACA, FCPRA and IATC channel assignment algorithms. By using less number of radio interfaces network leads toward lower co-channel interference and power consumption. 


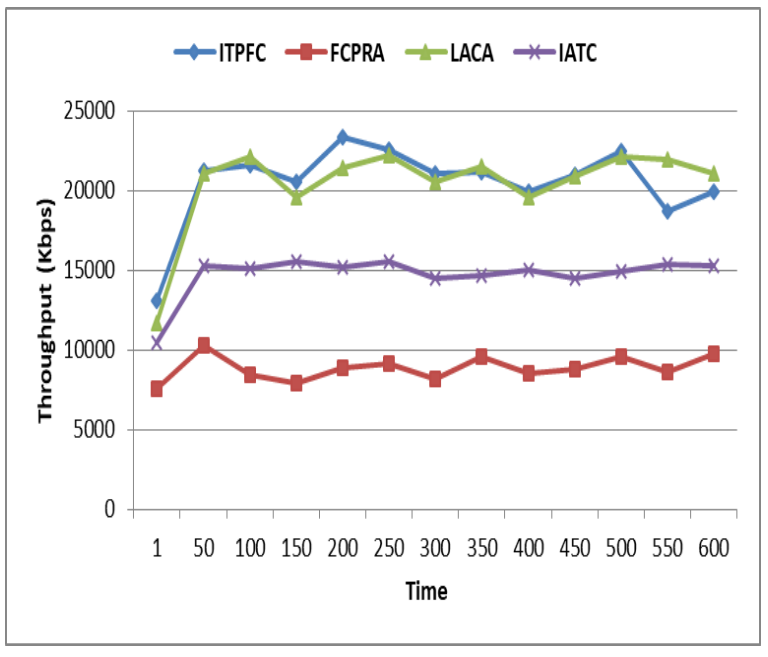

Figure 3. Throughput comparision for 40 nodes

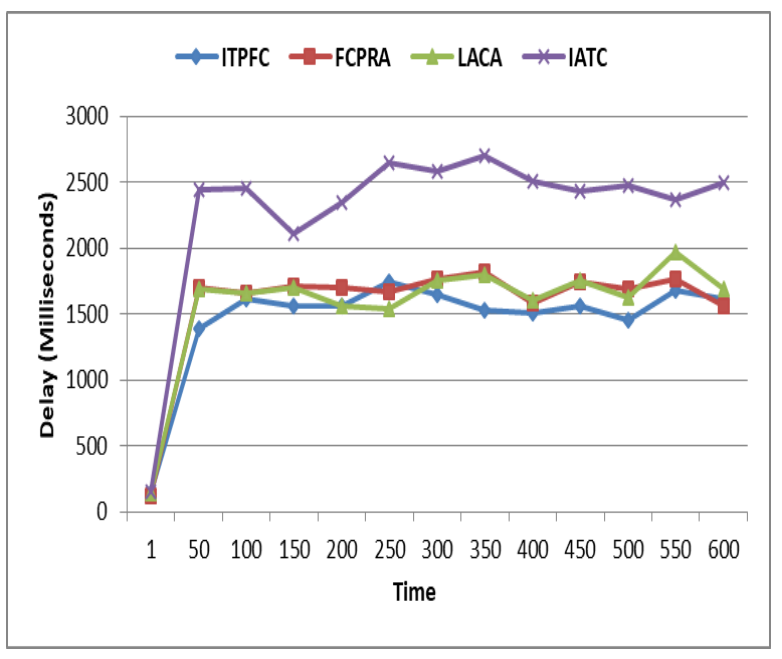

Figure 4. Delay comparision for 40 nodes

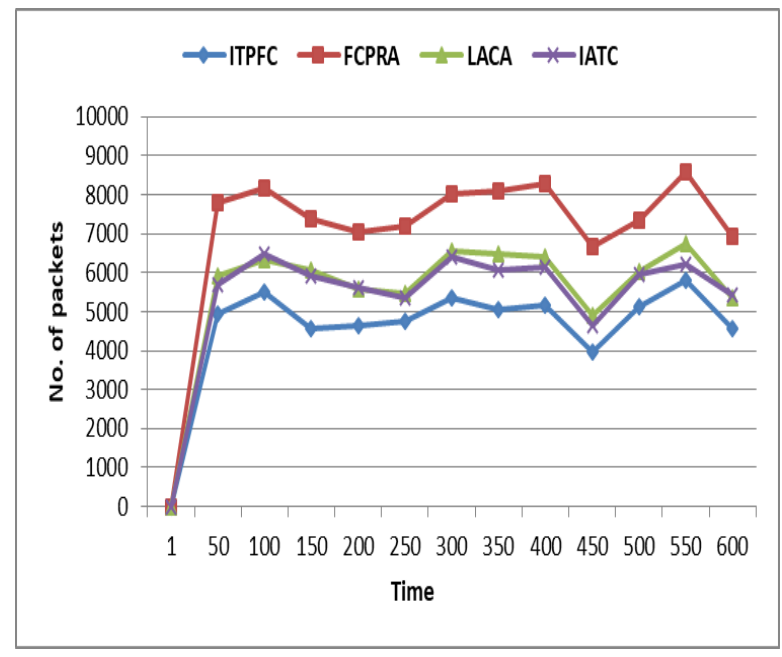

Figure 5. Packet loss comparision for 40 nodes

Table 2. No. of channels used

\begin{tabular}{ccccc}
\hline & ITPFC & IATC & LACA & FCPRA \\
\hline No. of Off Radios & 56 & 45 & 51 & 29 \\
\hline
\end{tabular}

\subsection{Results comparison of 50 nodes network}

Here, the results are compared for network of 50 nodes in terms of throughput, delay, packet loss and number of channels used per node as shown in Figures 6, 7,8 and Table 3. It is shown that the results of ITPFC Channel Assignment algorithm are marginally comparable in throughout and packet loss as compared to LACA and IATC. It provides suitable link capacity to transfer the data, so it has upper hand in results. The proposed algorithm provides better results in terms of delay and number of channels used per node as compared to FCPRA, LACA and IATC. In ITPFC 65 numbers of radio interfaces are off while in IATC, LACA and FCPRA 59, 60 and 23 number of radios are off respectively. Less number of radios used in network proves that the network has less co-channel interference and lower power consumption. Hance, ITPFC channel assignment algorithm works well and provides better network performance. 


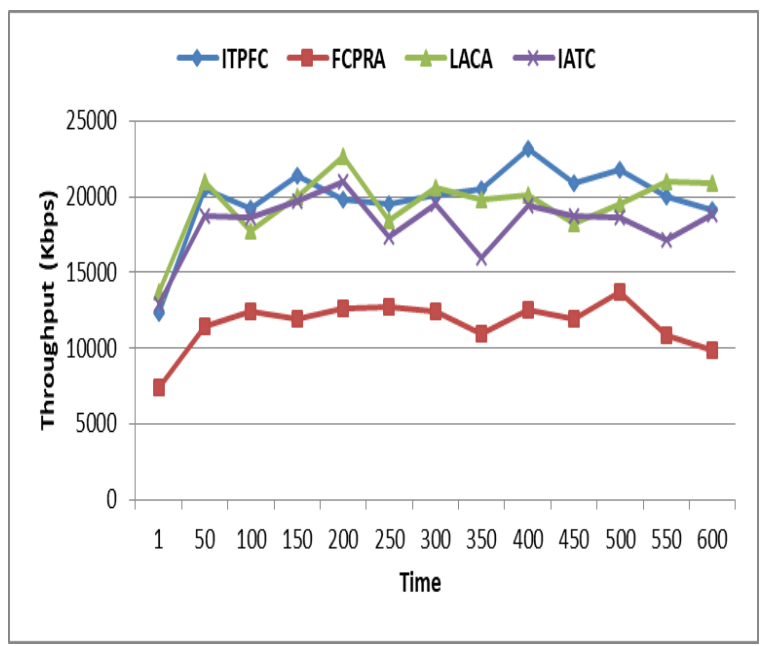

Figure 6. Throughput comparision for 50 nodes

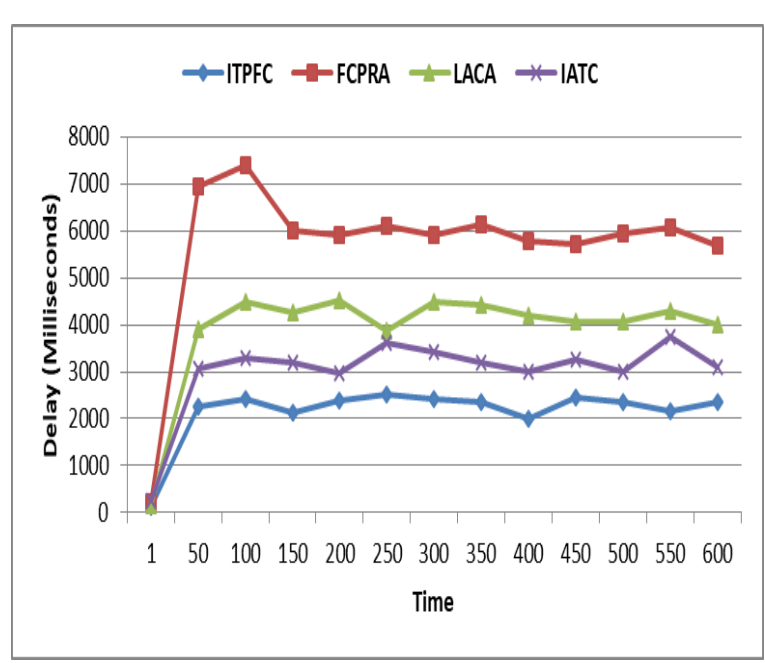

Figure 7. Delay comparision for 50 nodes

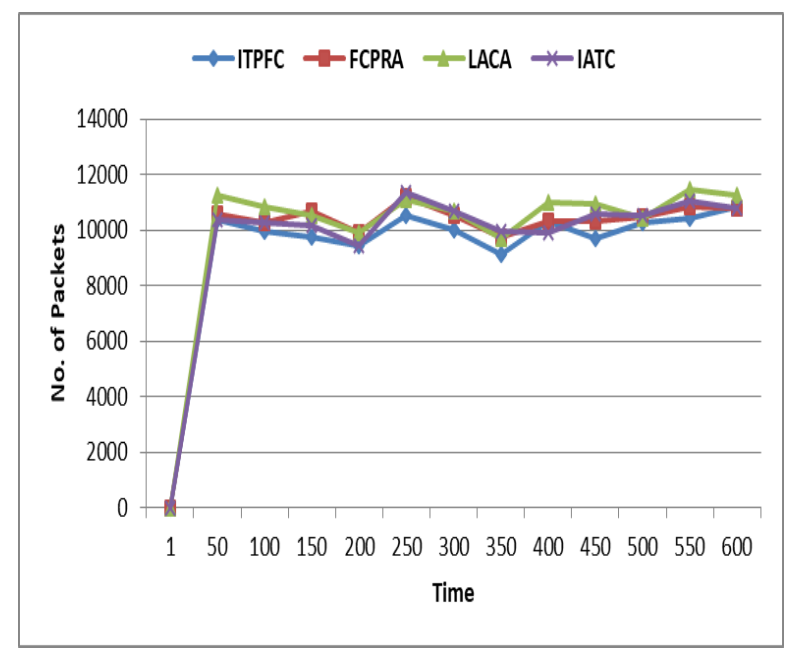

Figure 8. Packet loss comparision for 50 nodes

Table 3. No of channels used

\begin{tabular}{ccccc}
\hline & ITPFC & IATC & LACA & FCPRA \\
\hline No. of Off Radios & 65 & 59 & 60 & 23 \\
\hline
\end{tabular}

\section{CONCLUSION}

In this paper, Interference Aware, Topology, Power and Flow Control (ITPFC) Channel Assignment algorithm has been proposed for MRMC-WMN. The performance of the proposed algorithm has been evaluated for 40 and 50 nodes networks. The results obtained from these networks show the consistent performance in terms of throughput, delay, packet loss and number of channels used per node. It has been found that the ITPFC Channel Assignment algorithm used less number of radio interfaces to setup the network links as compared to LACA, FCPRA and IATC channel assignment algorithms. Due to this co-channel interference and power consumption of network have been reduced. Thus, the proposed ITPFC Channel Assignment algorithm has obtained better performance as compared to existing channel assignment algorithms. 


\section{REFERENCES}

[1] V. C. Gungor, et al., "Mesh Networks: Architectures and Protocols," Springer Science Business Media, New York, USA, chap1, pp. 1-27, 2008.

[2] K. S. Selvan, and B. Lakshmidhevi, "AProfessional QoS Provisioning In The Intra Cluster Packet Level Resource Allowance For Wireless Mesh Networks," International journal on applications of graph theory in wireless ad hoc networks and sensor networks, vol. 5, no. 1, pp. 1-10, 2013.

[3] N. Kaur, J. S. Saini, "Performance enhancement of 802.11 based wireless Mesh Network by using multi- radio multi-channel," In Proceeding of IEEE International Conference on Green Computing, Communication and Conversation of Energy, India, pp. 71-76, 2014.

[4] S. Avallone, et al., "A channel and rate assignment algorithm and a layer-2.5 forwarding paradigm for multi-radio wireless mesh networks," IEEE/ACM Transactions on Networking, vol. 17, no. 1, pp. 267-280, 2009.

[5] A. U. Chaudhry, et al., "On the impact of interference models on channel assignment in multi-radio multi-channel wireless mesh networks," Ad Hoc Networks, vol. 27, pp. 68-80, 2015.

[6] J. J. Galvez, and P. M. Ruiz, "Efficient rate allocation, routing and channel assignment in wireless mesh networks supporting dynamic traffic flows," Ad Hoc Networks, vol. 11, no. 6, pp. 1765-1781, 2013.

[7] J. J.Galvez, and P. M. Ruiz, "TCP flowaware Channel Re-Assignment in Multi-Radio Multi-Channel Wireless Mesh Networks," In the Proceeding of Eighth IEEE International Conference on Mobile Ad-Hoc and Sensor Systems, Spain, pp. 262-271, 2011.

[8] J. S. Saini, and B. S. Sohi, "Interference Aware, Topology, Power and Flow Control Channel Assignment Algorithm for Multi-Radio Multi-Channel Wireless Mesh Networks," International Journal of Computer Sciences and Engineering, vol. 6, no. 8, pp. 939-947, 2018.

[9] A. Raniwala, et al., "Centralized channel assignment and routing algorithms for multi-channel wireless mesh networks," ACM SIGMOBILE Mobile Computing and Communications Review, vol. 8, no. 2, pp. 50-65, 2004.

[10] S. Avallone, et al., "A new channel, power and rate assignment algorithm for multi-radio wireless mesh networks," Telecommunication Systems, vol. 51, no. 1, pp. 73-80, 2012.

[11] J. Tang, et al., "Interference-aware topology control and QoS routing in multi-channel wireless mesh networks," In Proceeding of 6th ACM international symposium on Mobile ad hoc networking and computing (MobiHoc '05), USA, pp. 68-77, 2005.

[12] J. Wang, and W. Shi, "Joint multicast routing and channel assignment for multi-radio multi-channel wireless mesh networks with hybrid traffic," Journal of Network and Computer Applications, vol. 80, pp. 90-108, 2017.

[13] C. Houaidia, et al., "Inter-flow and intra-flow interference mitigation routing in wireless mesh networks," Computer Networks, vol. 120, pp. 141-156, 2017.

[14] J. Kim, et al., "Distributed Channel Assignment Algorithm Based on Traffic Awareness in Wireless Mesh Networks," Wireless Personal Communications, vol. 95, no. 4, pp. 4983-5001, 2017.

[15] T. Yoshihiro, and T. Noi, "Collision-free channel assignment is possible in IEEE802.11-based wireless mesh networks," In proceeding of IEEE Wireless Communications and Networking Conference WCNC, USA, 2017.

[16] S. Choi, and J. H. Park, "Minimum Interference Channel Assignment Algorithm for Multicast in a Wireless Mesh Network," Sensor, vol. 16, no. 12, pp. 1-15, 2016.

[17] A. F. Tandjaoui, and M. Kaddour, "A joint power control, time-sharing and routing scheme to minimize spectrum utilization in wireless mesh networks," Journal of High Speed Networks, vol. 22, no. 3, pp. 205-221, 2016.

[18] A. Ouni, et al., "Energy and throughput optimization of wireless mesh networks with continuous power control," IEEE Transactions on Wireless Communications, vol. 14, no. 2, pp. 1131-1142, 2015.

[19] M. Shojafar, et al., "Improving Channel Assignment in Multi-radio Wireless Mesh Networks with Learning Automata," Wireless Personal Communications, vol. 82, no. 1, pp. 61-80, 2015.

[20] J. Wang, et al., "On channel assignment for multicast in multi-radio multi-channel wireless mesh networks: A survey," China Communications, vol. 12, no. 1, pp. 122-135, 2015.

[21] D. Wu, "Joint multi-radio multi-channel assignment, scheduling, and routing in wireless mesh networks," Wireless Networks, vol. 20, no. 1, pp. 11-24, 2014.

[22] S. Avallone, "An energy efficient channel assignment and routing algorithm for multi-radio wireless mesh networks," Ad Hoc Networks, vol. 10, no. 6, pp. 1043-1057, 2012.

[23] N. Ahmad, et al., "Enhanced topology-controlled interference-aware channel assignment for multi-radio multichannel wireless mesh networks," In proceeding of Wireless Days, Canada, pp. 1-6, 2011.

[24] A. A. Franklin, et al., "On the end-to-end flow allocation and channel assignment in multi-channel multi-radio wireless mesh networks with partially overlapped channels," Computer Communications, vol. 34, no. 15, pp. 1858-1869, 2011.

[25] H. Friis, "A note on a simple transmission formula," In proceeding of the I.R.E. and Waves and Electrons, vol. 34, no. 5, pp. 254-256, 1946.

[26] D. Tse, and P. Viswanath, Fundamental of wireless communication, Cambridge University Press, USA, chap5, pp. 195-265, 2005. 


\section{BIOGRAPHIES OF AUTHORS}

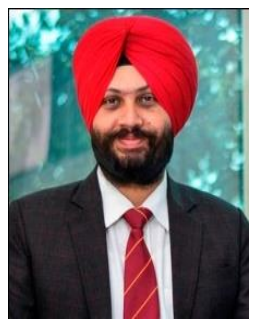

Jatinder Singh Saini Has received his degrees of B.Tech in 2005 \&M.Tech in 2011 from Punjab Technical University, Jalandhar, Punjab, and pursuing Ph.D. in the discipline of Computer Science \& Engineering from the same University. He is having experience of 13 years. His research areas of interest are wireless communication, computer networks and wireless mesh networks.

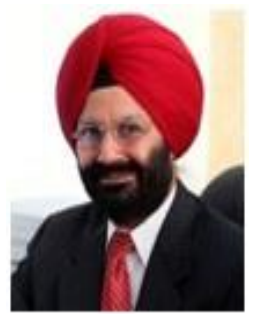

B.S. Sohi Has received his degrees of B.Sc. Engineering, Master of Engineering \& Ph.D. in Electronics in years 1971, 1981, 1992 from Panjab University, Chandigarh. He is the Ex- Director of UIET, Panjab University, Chandigarh and presently he is working as Director at Chandigarh Group of Colleges, Mohali, Punjab. He is having experience of 35 years in teaching and administration. He has 105 research publications in various fields. His areas of research are wireless networking, computer networking and wireless sensor networks. 